\title{
Simulation Studies of Distributed Two-phase Locking in Distributed Database Management Systems
}

\author{
S. Vasileva, A. Milev
}

Key Words: Simulation models; concurrency control; distributed database; distributed transactions; distributed 2PL.

\begin{abstract}
This paper considers algorithms simulating the implementation of distributed two-phase locking (2PL) protocols in distributed database systems and simulation results. It describes specifically the simulations of two-version $2 P L$ and $2 P L$ with integrated timestamp ordering mechanism. Integrated modelling algorithms for deadlock avoiding are suggested in the paper: twoversion architecture of database and timestamp ordering strategy "wait-die". The results of the simulations of these two variants of the 2PL method at different scales of the networks for data transmission and at different intensities of inflow transactions are also presented. Modelling algorithms are developed by means of the system for simulation modelling GPSS World Personal Version.
\end{abstract}

\section{Introduction}

Many of information systems and systems management have a distributed structure meeting the requirements of the relevant application areas much better than centralized ones. All this is valid for the distributed database (DDB) systems and Distributed Database Management System (DDBMS). A major problem in these systems is concurrency control to general data parallel executed transactions [2]. Concurrency control techniques are divided into: Locking, Timestamp ordering and Optimistic strategies -Validation check up according to $[4,5,6$, etc.]. In the last two methods were obtained better retention of transactions in the system saturated by conflicts. The problem is solved by using the method of Two-phase locking - 2PL. The deadlocks of transactions may arise at their processing in systems used 2PL method and in DDBMS at different sites of the distributed system.

The basic methods for avoiding deadlock transactions are: multiversion (two-version) architecture of data in database [3] and embeded timestamping (TS) algorithm, working in one of two strategies: "wait-die" and "wound-wait" described in $[4,5]$, etc.

The problems in the application of 2PL method in DDBMS and avoiding transactions of deadlocks require testing. Methods 2PL in DDBMS are four varieties: Centralized 2PL, Primary Copy 2PL, Distributed 2PL and Voting 2PL. In the paper we consider algorithms, modelling the protocol for Distributed 2PL [4,5]. The general purpose system simulation (GPSS World) was chosen for development environment modelling algorithms Distributed Two- version 2PL (2V2PL) and 2PL with integrated TS method (wait-die).

The studied sources on simulation ([7], etc.), convince us in the benefits of the environment GPSS World for simulation systems for processing transactions, such as DDBMS. In front of the authors is standing the problem to study transaction concurrency control in DDBMS, in which the data are subject of fragmentation and replication on system nodes.

\section{DDBMS and Distributed} 2PL. GPSS Model

As a base for the formal model we use the structure suggested in [2]. The structure is used in the architecture of the moderm DDBMS that work on Distributed protocols (2PL, TSO, etc.) The modules which participate in the transaction processing are depicted with circles and the necessary data with rectangles. Figure 1 shows the model of the structure of the DDBMS, in which concurrency control is executed on distributed 2PL. We consider the following basic DDBMS components that operate in every site, such as transaction source $\left(S_{i}\right)$, transaction coordinator $\left(T C_{i}\right)$, System Catalogue $\left(S C_{i}\right)$, Data Manager $\left(D M_{i}\right)$, Lock Manager $\left(L M_{i}\right)$, Lock Table $\left(L T_{i}\right)$.

Transaction coordinator includes the component for data communication. It divides the global transaction to sub-transactions, based on the Global System Catalogue information. If some transaction requires renovation of replicated data element the coordinator has to request a specific lockings for all its copies and after that to release these lockings. In order to read renovated element the coordinator can choose one of the existing copies.

Transaction Manager controls the execution of running transactions of diferent apllications and interacts with the Scheduler;

Lock Manager (Scheduler or concurrency manager) is responsible for realization of chosen protocol to control parallel transaction processing in the system. The Lock manager checks the validation of incoming requests for data element lockings. It is based on current locking states of them. If the locking is validated LM sends a message to the site-initiator to confirm that requested locking is granted. In other case the request is set in a queue to wait for proper processing.

LM, TM, and DM communicate via passing messages [4]. 


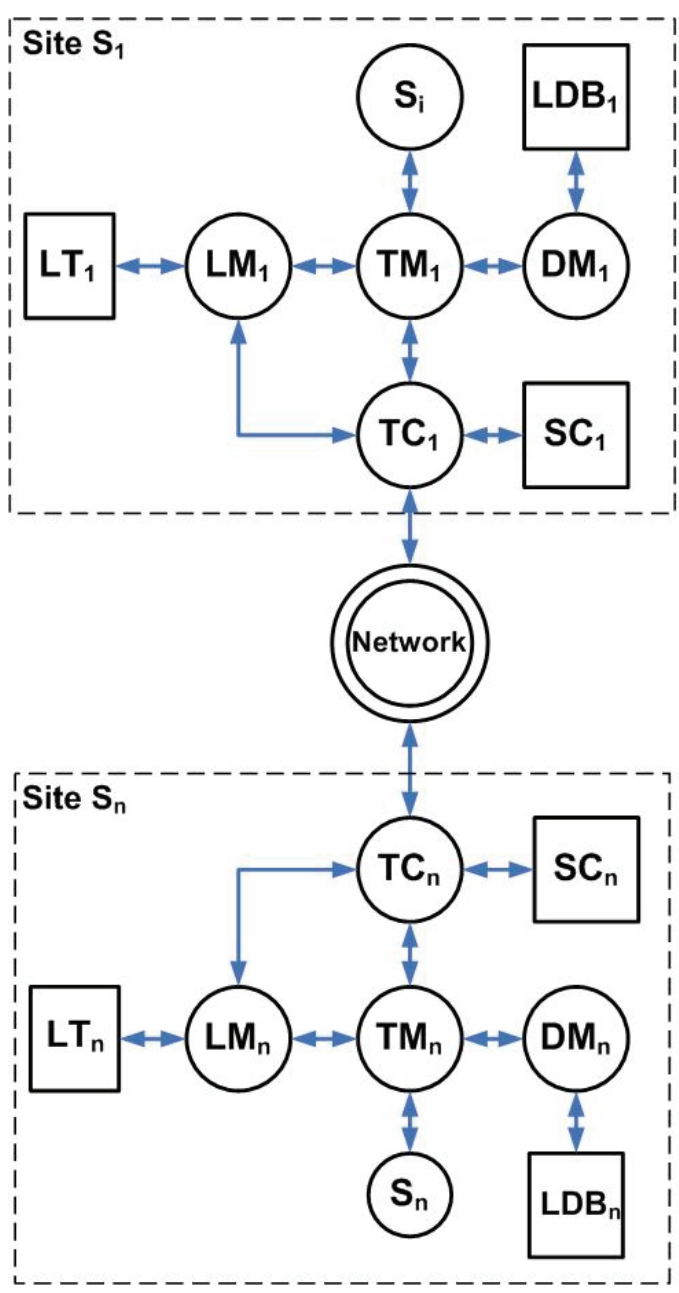

Figure 1. DDBMS structure at Distributed 2PL

\subsection{Distributed Two-Phase Locking of Distributed Transactions}

The summary modeling algorithm of transaction processing for simulation with Distributed 2V2PL is given at figure 2.

\subsection{GPSS Model of the Queuing System}

In the construction of probabilistic models of computer systems of different types and especially information systems for public use, the processing of user requests is described as service requests in a closed queuing system (QS). The number of service phases depends on the configuration of the test system. Some of these phases are: the number of QS, forming the network, the probability of transmission of requests from one phase to another, the number of parallel channels for service workers in phases, average times and laws of service requests phases distribution [1]. Furthermore, the structure of the model and the topology model depends a lot on the algorithm processing the requests of users in the system. For the network of QS in the model it is assumed that:

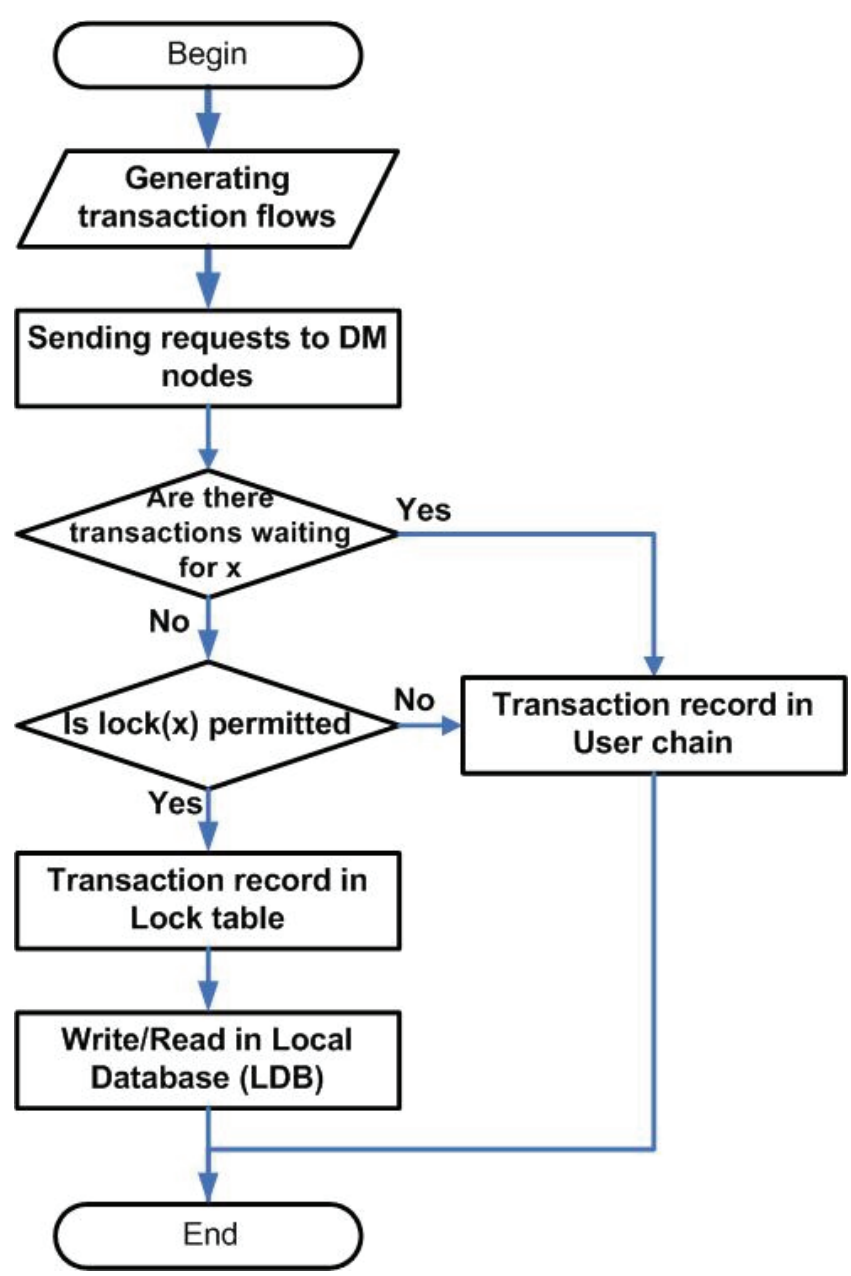

Figure 2. Summary modeling algorithm of Distributed 2V2PL

- The flow of the global transactions receipt in sites DDBMS is Poisson with parameter $\lambda$.

- The duration of service transactions has exponential distribution with parameter $\mathrm{m}$.

- Serving devices are arranged alternately in parallel channels.

- Discipline of service queues is „First Input - First Serviced".

- Incoming transaction does not leave the system until it has been serviced.

In our case we assume that transactions go to the sites on an exponential law. They are sent on parallel channels, recorded in certain incoming and outgoing buffers reach sites with the necessary data and transmitted to the recipient (leave the system).

\subsection{GPSS Model of Distributed 2PL of Distributed Transactions}

The main component in Database management system, carrying out the transaction concurrency control is the Scheduler. The Lock manager is used when 2PL protocol is 
working. When DDBMS works on Distributed 2PL protocol, the system has Lock managers for each site distributed by the system $[4,5]$, etc. We consider the models Distributed 2PL where each data element has two replicas. The model generates 6 streams GPSS transactions. Each GPSS transaction models a global transaction in DDBMS. Each stream is characterized by intensity $\lambda$ transactions per second.

A lot of transactions, which are managed by the relevant transaction managers $\mathrm{TM}_{1}-\mathrm{TM}_{\mathrm{k}}$ get in different sites $S_{1}-S_{k}$ of the distributed system. We consider the work of DDBMS on distributed 2PL protocol. Therefore the queries for locking the element $\mathrm{x}$ are sent directly to sitesexecutors, in whose local databases are stored copies to $\mathrm{x}$. A lot of local lock managers exist, responsible for locking the copy of the element $x$. Indications for incoming operations are as the follows: $r_{n}(x)-$ transaction with number $n$ $\left(T_{n}\right)$ which wants to read element $x ; w_{n}(x)-T_{n}$ recording the value of the element $x ; c_{n}-T_{n}$ successfully completes its operation and fixes; $a_{n}-T_{n}$ interrupts his performance and restarts.

The algorithms for Two-version two-phase locking for DDB are one of possible solutions for protocols of 2PL in DDBMS [4]. The permission of lock acceptance or its refusal is done by a lock manager, which works under the 2V2PL algorithm and consistency matrix, described in details in [4].

The Two-version 2PL protocol is described in details in [3], and the modeling algorithm is presented in [10].

The algorithm modeling Distributed 2PL with an integrated mechanism of TS (strategy "wait-die") is described in [9].

\subsection{Parameters of the Generated Transactions in Simulation Models}

The generated GPSS transactions are described by many parameters as the ones in $[9,10]$ with different values, such as Number of the transaction $(P 1)$, Number of the site generated by the transaction $(P 2)$, Length of the modeling of GPSS distributed transaction $(P \$ N e l)$, Number of elements (P\$El1, P\$El2), Type of lock (P\$Bl1, P\$Bl2).

The number of the transaction is the sum of System Numeric Attribute (SNA) MP2 (the difference between the relative time model and the contents of the second parameter of the GPSS transaction) and the number of the site.

The number of the site generated by the transaction is an integer from 1 to <the number of streams transactions $>$. In the presented models, <cash flows transactions $>=6$.

The length of the modeling of GPSS distributed transaction could accept 1 or 2 , selected with probabilities given by the function $F N \$ B r E l$, respectively 0.30 and 0.70 . The model assumes that the "long" transactions enter the system more often than the shorter.

The number of the first (second) element that the generated transaction will "read" or "updated". The value is chosen at random number uniformly distributed in the range [1, NumEl].

The type of lock applied to the first (second) element, processed by generated transaction could accept 1 (reading), 2 (update) or 3 (for recording), selected with probabilities given by the function FN\$LockTip1. Originally the values are set at $0.40,0.30$ and 0.30 respectively. The function for the second element FN\$LockTip2 originally is set at values $0.10,0.30$ and 0.60 , respectively.

In the parameters P\$CHTN1, P\$CHTS1 (P\$CHTN2, $P \$ C H T S 2$ ) are recorded, the number of previous transaction, blocked the element and the numbers generated by the site (by LT). It is permited if P\$B11=1 (P\$B12=1) when the transaction will only ,read" the element with number P\$El1 (P\$El2) and if the element is not available, but the lock is allowed.

The parameter $P 5$ could has value 0 , if the transaction is in the first phase - seizure of locks and a value of 1 if the transaction completes its work and must release the locks. In simulation of longer transactions, where it can take place deadlocks on transactions can be assigned a value of 2 to P5 of transaction, which would, needs to reboot.

The parameters $P 6, P 7, P 8$ and $P 9$ record the number of the site where the first/second copy $(P 6 / P 7)$ of first data element (P\$E11) and first/second copy $(P 8 / P 9)$ of second data element $(P \$ E l 2))$, is processed by the transaction $\mathrm{x}_{\mathrm{k} 1} \mathrm{El} 1, \mathrm{x}_{\mathrm{k} 2} \mathrm{El} 1, \mathrm{x}_{\mathrm{k} 3} \mathrm{El} 2, \mathrm{x}_{\mathrm{k} 4} \mathrm{El}$.

The parameter $P \$ v r$ is used in the model of $2 \mathrm{PL}$ with timestamping $-P \$ v r=1$ in the case that the lock of the element is not possible and the number of the GPSS subtransaction is smaller than the number of the transaction, which set the lock. According to timestamping mechanism strategy "wait-die" [4] if subtransaction does not continue and does not restart, it waits for the release of the elements in the user chain, whose number is the number of elements.

\subsection{Auxiliary Variables and Matrices, Used in Modeling Algorithms}

Besides the parameters of transactions in 2PL modeling algorithms shall also apply the following options of GPSS World:

Matrices. In the synthesized simulation models the matrices are used (MX\$LTA1 and MX\$LTA2) for modeling the lock tables in the sites executors for the first and second replica of each data element. Proper locking records of the replica are set. Each row of the table corresponds to the data element from DDB. Each matrix has the following columns:

- Type of the resulting lock the GPSS transaction blocked free data elements. This column, records the value parameter $P \$ B l 1$ or $P \$ B l 2$ of the same (depending on whether the element is the first or the second processed by transaction).

- Number the GPSS transaction blocked free element. This column records the value the $P 1$ parameter transaction, which placed the lock in the first column.

- Number of the site - initiator the GPSS transaction blocked first element (the value of parameter P2 of the transaction, borrowed the element in the lock table). 
- Number of the GPSS transaction, received a shared lock element, blocked by another "reading" transaction in previous moment. This column records the value the P1 parameter of the transaction, whose request for "reading" is compatible with the shared lock set by the first column.

- Number of the GPSS transaction, received an interlock "record" elements blocked by "reading" transaction in previous moment. This column records the value of transaction parameter ( $\mathrm{P} 1)$, whose request for "record" is compatible with the shared lock, set by the first column 2V2PL modeling algorithm.

In the synthesized simulation models are used also and two matrices ( $M X \$ R A Z S T$ and $M X \$ R A Z D E V)$ to set the mean and standard deviation of the retention time of he transactions in the transmission of messages between the nodes of the distributed database system modeled for communication costs.

Variables. In the simulation models primarily are used the following variables.

The variables V\$ElemN1 (V\$ElemN2) contain calculated random selection numbers of the first (second) element that will process the transaction. In the statements which calculates variable ElemN1 and the ElemN2 is involved and the random number generator RN2.

The variables $V \$ R A Z R B L 1$ (V\$RAZRBL2) contain calculation of the admissibility of the first (second) lock element processed by the transaction. The value is the product of the parameter P\$Bl1 (P\$Bl2) of the GPSS transaction requesting the lock and the value in the first column of the matrix LTA1/LTA2.

Cells. In the synthesized simulation models are used the following cells serving as different counters.

The counter X\$BROITR keeps total number of generated GPSS transactions during the modeling.

The counter $X \$ Z A V T R$ contains total number of served transactions leaving the model.

The counters X\$BROITR1 (X\$BROITR2) keep the total number of generated transactions, processing one (two) data element;

The counters X\$ZAVTR1 (X\$ZAVTR2) contain number of served transactions with length 1 (2) leaving the modeled system.

\section{Simulation Results}

The studies over the simulation models, are evaluated in the GPSS World environment in order to collect, summarize and analyse the results of the modelling algorithm of Centralized 2V2PL and modelling algorithm of Distributed 2PL with an integrated mechanism of TS in DDB. Calculation of the main characteristics of service transactions DDBMS are given. These characteristics are Throughput (TP), Service Probability (SP) and Response time (RT). Throughput of one system is calculated such as number of requests serviced per unit time [7]. This indicator is calculated by the formula (1)

$$
X=\frac{N_{c}}{T_{n}}
$$

where $N_{C}$ is the total number of the committed transactions (in the synthesized models it is the value of the cell $X \$ Z A V T R)$, for the monitoring period of the system $-T_{n}$ (modeling time for different startups of the modeling algorithm).

The developed modeling time in the GPSS World algorithms is set in milliseconds. All streams transactions are received upon an exponential law with a variable for different studies with an average length of the interval. We consider 6 streams generated by GPSS transactions. The model include 6 sites in distributed database system, for which global transactions have Poisson law in all modeling algorithms.

The diagram of figure 2 presents the results of simulations of Throughput Distributed 2PL algorithms at the same intensities of input flows depending on the monitoring period (in seconds). The graph marked with a thin blue dashed line (2V2PL) and the graph indicated by a thick black line and square markers (2PL TSwd) present 6 streams with an average intensity 4,17 tr/s (minimum load - intensity cumulative flow $25 \mathrm{tr} / \mathrm{s}$ ). The graph marked with thin black line and asterisks (2V2PL) and in the graph illustrated by dashed lines with triangular markers (2PL TSwd) are 6 streams, with an intensity of $8,33 \mathrm{tr} / \mathrm{s}$ (average load - intensity of the aggregate stream $50 \mathrm{tr} / \mathrm{s}$ ) for each of them. The graph indicated by the thin dotted line (2V2PL) and the graph indicated by a thin blue line (2PL TSwd) are 6 streams of medium intensity $16,67 \mathrm{tr} / \mathrm{s}$ (maximum load - intensity of aggregate stream $100 \mathrm{tr} / \mathrm{s}$ ).

The diagram in figure 3 shows Throughput for both models at three operating modes of load increased with the growth of the model during and after a certain time (5 minutes after the start of operation of the system). The occurrence of stationary mode reaches a maximum value and does not change with time.

Service probability factor or completeness of service transactions serves to assess the dynamic properties of DDBMS. The probability of service $P_{s}$ of distributed transactions is calculated by the formula $(2)$ [7]

$$
\text { (2) } P_{s}=\frac{N_{c}}{N_{g}} \text {, }
$$

where $N_{C}$ is the total number of fixed transactions (cell value of $X \$ Z A V T R$ after modeling, and $N_{g}$ is the total number of transactions generated for the same period of time (cell value of $X \$ B R O I T R$ after modeling).

Figure 4 shows the results for the probability of service of distributed transactions at simulation algorithm Distributed 2V2PL and Distributed 2PL with an integrated mechanism of timestamping at the same intensities of inflows (figure 4).

The results obtained for the average Response time 


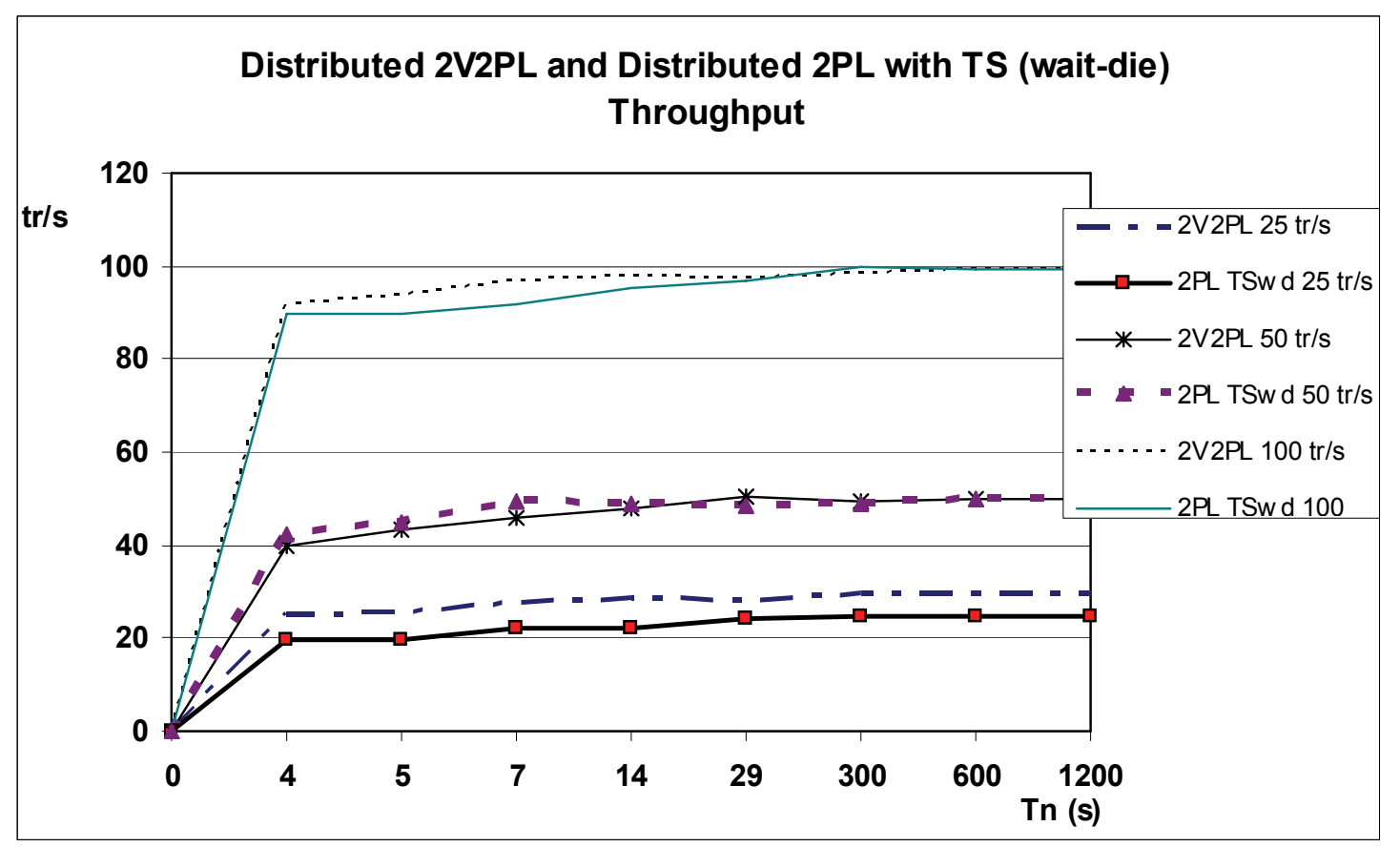

Figure 3. Throughput of the systems

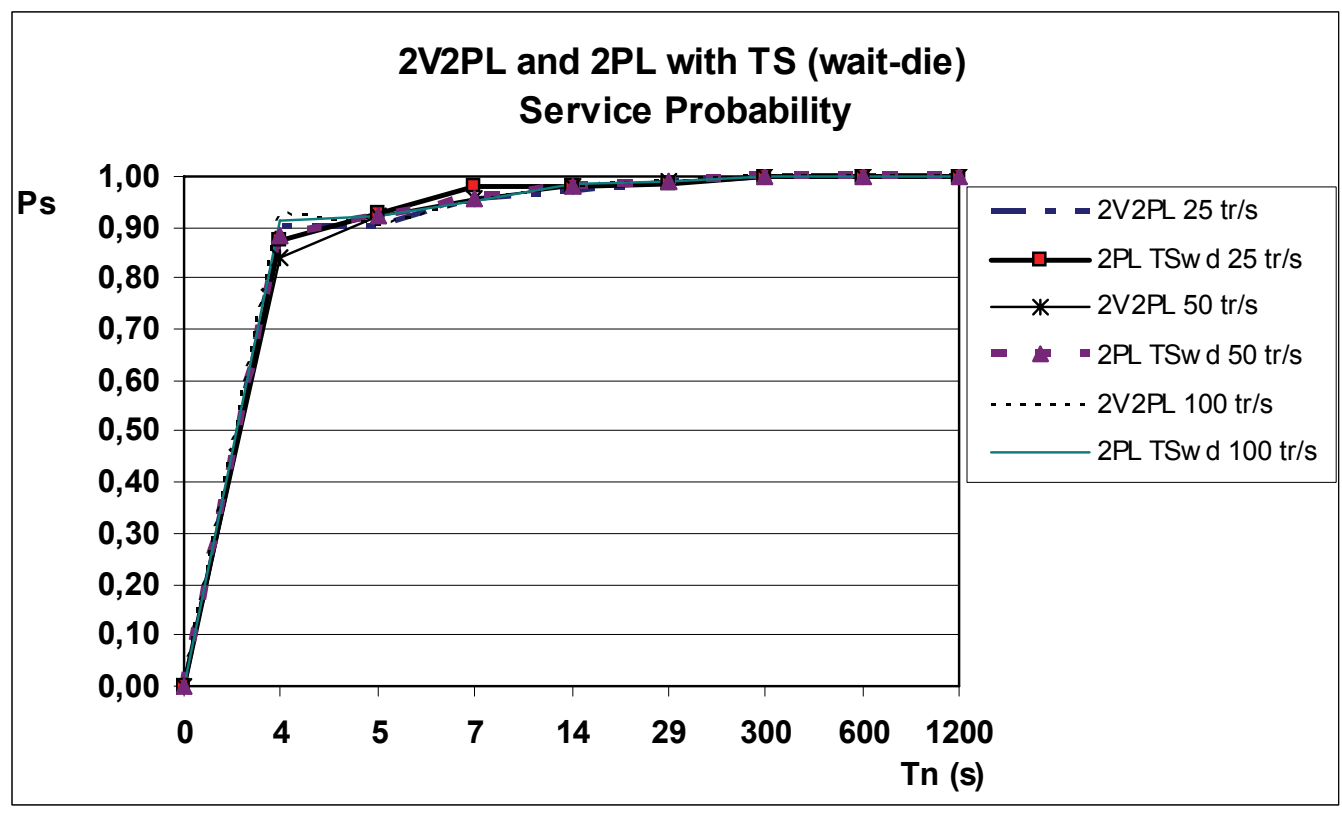

Figure 4. Service probabilities in the 2V2PL model and the 2PL with TS "wait-die" model

for modeling of Distributed 2V2PL and Distributed 2PL with TS "wait-die" are given in table 1. The data are taken automatically from the generated reports by GPSS World after the end of each simulation. In the codes of the models is set a GPSS table DaTable, which records the "residence time" (SNA of GPSS - MI) model of each GPSS transaction. Furthermore, the Response Time could be fixed and from the table of queues generated in the report of modeling algorithm implementation. The queues are realized by GPSS blocks QUEUE < name of a queue $>$ - entry in the respec- tive queue and DEPART < name of a queue $>$ - exit from the respective queue. In the report of the queue GPSS World model displays the following statistics for each one: maximum length of the queue, average length of the queue, number of entries in the queue, average time of residence in the queue of all transactions, etc.

In all models values of its parameters are assigned after the generation of each transaction. These parameters are quantity of processed data elements, the numbers of the elements that will be processed, read or updated, the num- 


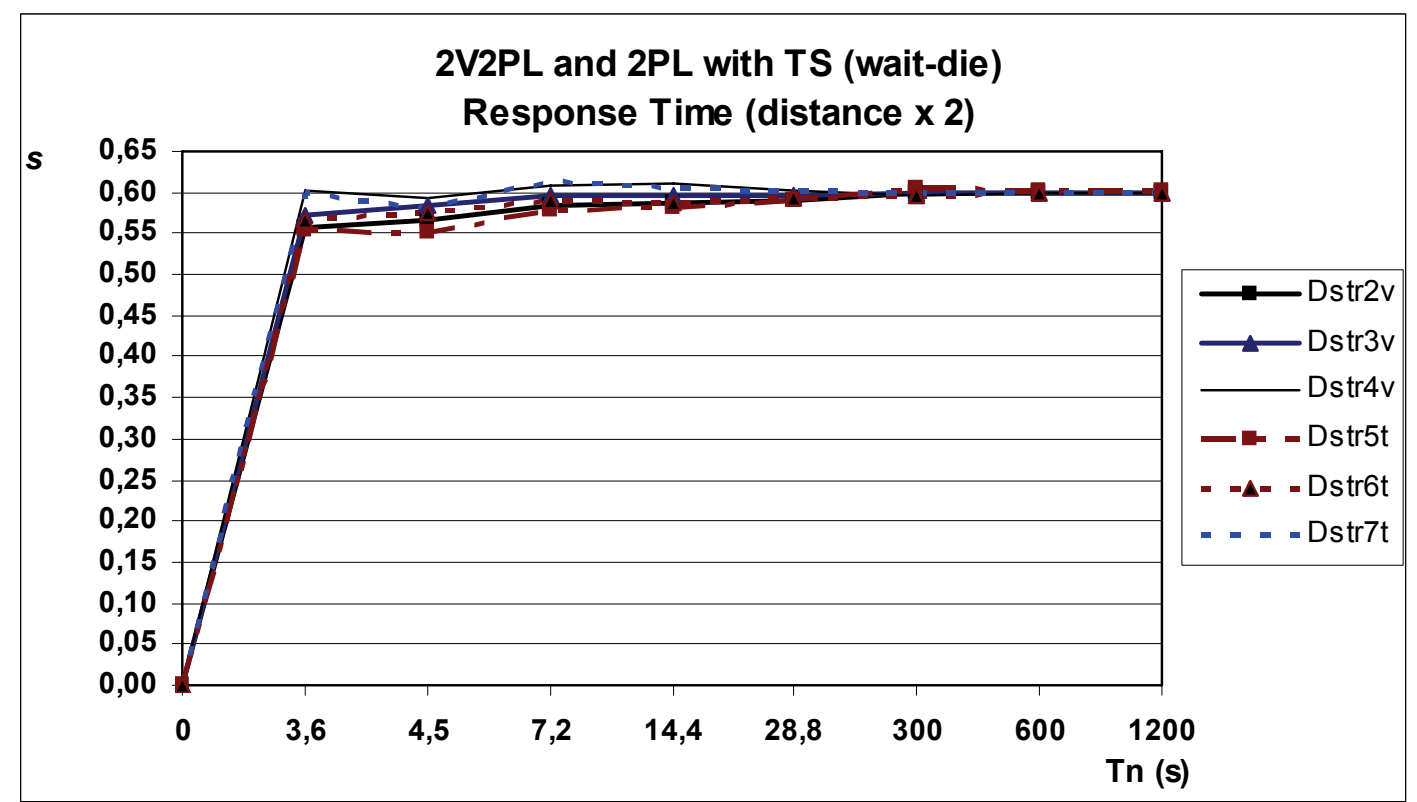

Figure 5. Response time of transactions in model systems at different intensities of inflows

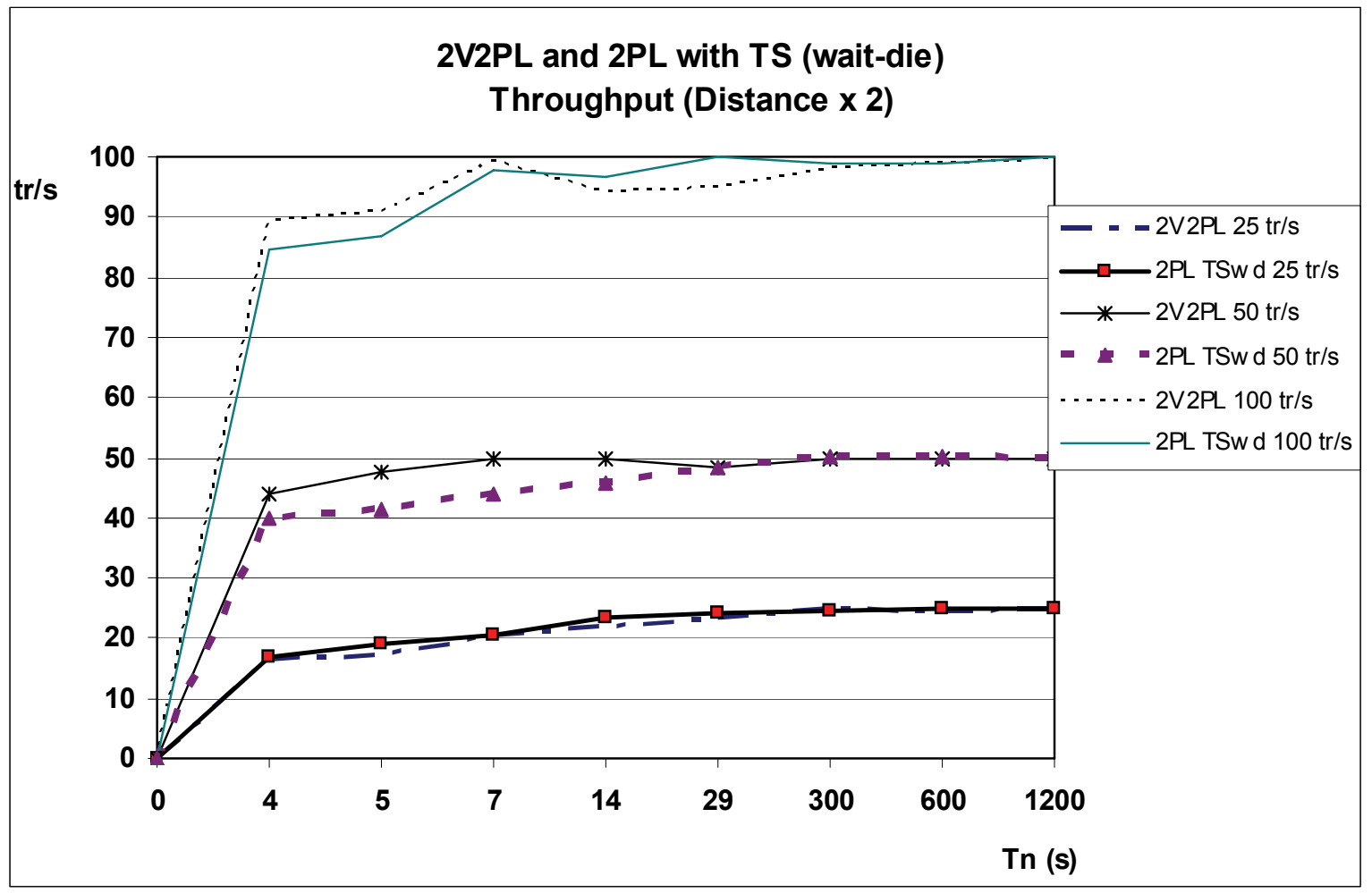

Figure 6. Throughput in the models at doubled distance between the sites in the system 


\section{The Average Response Time in the models of Distributed 2V2PL and Distributed 2PL with built timestamping (in seconds)}

\begin{tabular}{|c|c|c|c|c|c|c|}
\hline Tn & Dstr2v & Dstr3v & Dstr4v & Dstr5t & Dstr6t & Dstr7t \\
\hline $\mathrm{s}$ & $100 \mathrm{t} / \mathrm{s}$ & $50 \mathrm{tr} / \mathrm{s}$ & $25 \mathrm{tr} / \mathrm{s}$ & $100 \mathrm{t} / \mathrm{s}$ & $50 \mathrm{tr} / \mathrm{s}$ & $25 \mathrm{tr} / \mathrm{s}$ \\
\hline 3,6 & 0,557 & 0,571 & 0,601 & 0,553 & 0,567 & 0,600 \\
\hline 4,5 & 0,567 & 0,584 & 0,594 & 0,550 & 0,574 & 0,582 \\
\hline 7,2 & 0,583 & 0,597 & 0,609 & 0,578 & 0,590 & 0,615 \\
\hline 14,4 & 0,586 & 0,595 & 0,610 & 0,582 & 0,588 & 0,606 \\
\hline 28,8 & 0,589 & 0,595 & 0,603 & 0,590 & 0,594 & 0,603 \\
\hline 300 & 0,600 & 0,599 & 0,597 & 0,604 & 0,597 & 0,599 \\
\hline 600 & 0,598 & 0,600 & 0,599 & 0,601 & 0,600 & 0,600 \\
\hline 1200 & 0,598 & 0,598 & 0,599 & 0,601 & 0,599 & 0,600 \\
\hline
\end{tabular}

bers of sites where are the copies of the elements that will be processed, etc. After setting the values of "common" for each transaction parameter, the transaction enters into the queue TOTALTIM. Once the transaction is fixed, exits the TOTALTIM queue, in which the transaction "submit" the information about itself in the automatically collected statistic by GPSS before leaving the model.

The data in the table are obtained by the following intensity:

- In Column 2 (2V2PL) and Column 5 (2PL TSwd) each of the six flows has an average interval of receiving the transactions 60 model units. The intensity of the aggregate flow is the highest of those surveyed - $100 \mathrm{tr} / \mathrm{s}$ (in the general case, the transaction ends her performance later then the generation of the next transaction in the stream).

- In Column 3 (2V2PL) and Column 6 (2PL TSwd) each of the six flows has a mean interval of placing the model units 120 transactions. The intensity of the aggregate flow is the average value in the surveyed $-50 \mathrm{tr} / \mathrm{s}$ (in each transaction, the flows have the opportunity to leave the served a little after the generation of the next transaction).

- In Column 4 (2V2PL) and Column 7 (2PL TSwd) each of the six fluxes has a mean interval of placing the model units 240 transactions. The intensity of the aggregate flow is the lowest of those surveyed $-25 \mathrm{tr} / \mathrm{s}$ (in each transaction, the flows have the opportunity to leave the served, before the generation of the next transaction).

The diagrams of figure 5 show graphical form of the data from the table. It could be seen that the graphics of RT measurements have a rapid increase in the beginning, a slow growth after that and stationary mode. Furthermore, the graphs of the six simulations hardly differ, which specifies the advantages of the Distributed 2PL. It could be explained by the specificity of the protocol. If the transaction, which wants locking the element is older than the transaction occupied by the element, which has a smaller number, it restarts with a reservation number. So, the next race for locking elements would be with the smallest number and will receive the locking of the elements, to accomplish after the read/write operation. This is the reason why the lock manager service delays according to the site-initiator. It increases the time of service of the transaction.

The calculated throughput (1) by substituting the fixed one in the receiving reports values of the cell $X \$ Z A V T R$ is given on figure 6. The intensity of inflows transactions are the same as the graphs of figure 3. It was changed only for the matrix of distances MX\$RAZST. The values in it are increased twice compared to the models whose results are reported in the graphs of figure 3.

By altering the values of cells in the matrix MX\$RAZST we can conduct research whether and how the gaps in the net, impact on TP, SP and other performance indicators of concurrency control algorithms. For example, by increasing the cell values of the matrix MX\$RAZST five times, the values of the TP are obtained from the simulation reports of the Distributed 2V2PL (graphs on figure 7) and the Distributed 2PL with TS "wait-die" at the same intensities of inflows as in figure 3.

Increasing the distance between the sites in DDBMS does not affect the throughput of the distributed system, even at the maximum load. It is confirmed by the graphs of figure 8 , which are obtained by increasing the values of the cells in the matrix MX\$RAZST 8 times compared with the values for the simulations shown in figure 3 .

Another indicator of the performance of concurrency control algorithms is Frequency distribution of RT of transactions. The diagrams of Frequency distribution of RT are built automatically by the formulated in the GPSS model tables. The histogram of Frequency distribution of RT in modeling Distributed 2V2PL is demonstrated in figure 9 with total intensity of the input streams $100 \mathrm{tr} / \mathrm{s}$ (maximum load) and observation time 28.8 seconds. A histogram is generated automatically by GPSS World on table RT distribution DATABLE, whose values are given in the simulation report.

The histogram of Frequency distribution of RT in modeling Distributed 2PL with timestamping (wait-die) is demonstrated in figure 10 for total intensity of the input streams $100 \mathrm{tr} / \mathrm{s}$ (maximum load on the system) and observation time 28,8 seconds (as in figure 9).

The tables of Frequency distribution of RT besides that serve comparative analysis of concurrency control algorithms, serve also to assess the reliability of modeling algorithms by comparing with the template chart of Frequency distribution of RT cited in [8]. Similarly, it could be compared with the template graphics and charts for throughput of figure 3, figure 6 and figure 7. 


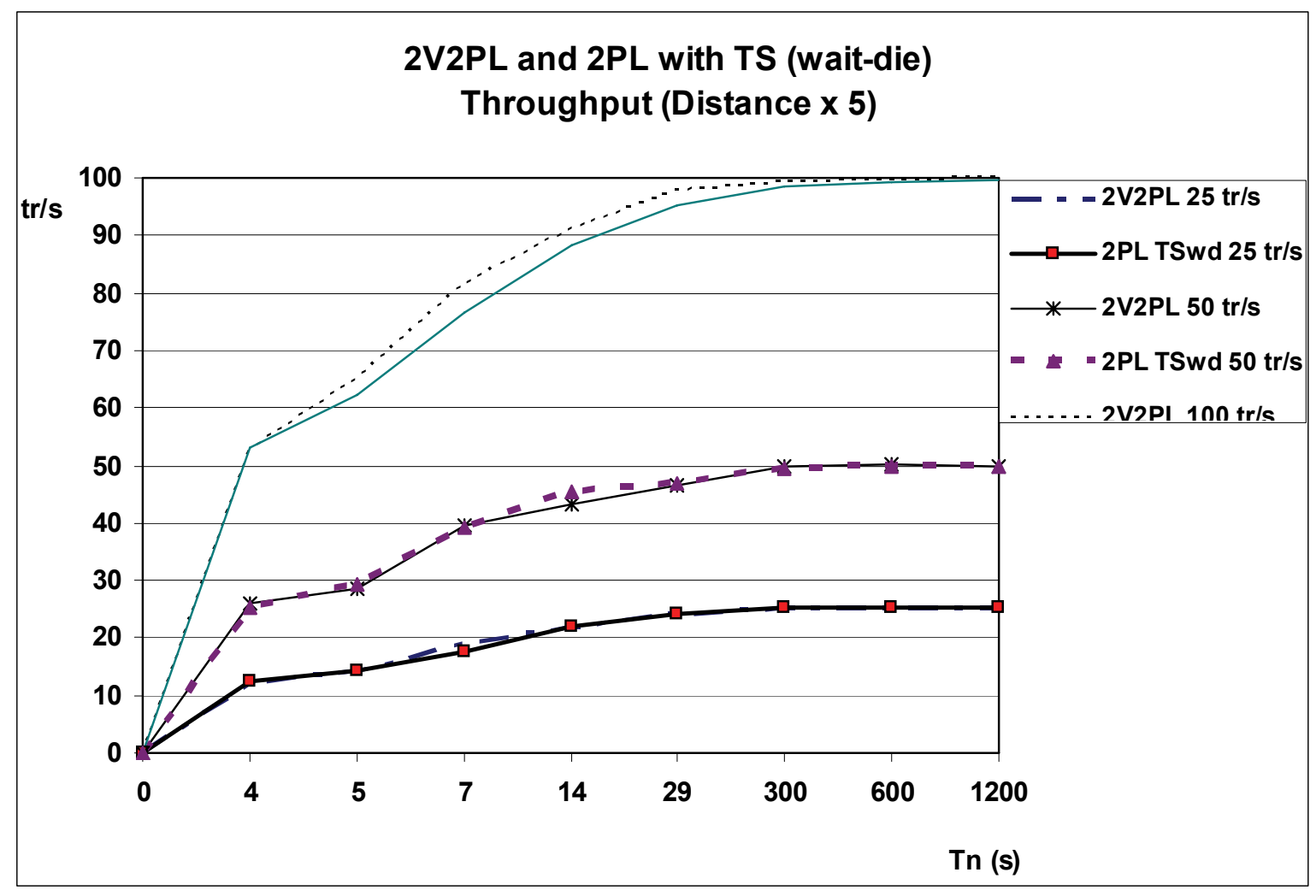

Figure 7. Throughput of the system (distance x 5)

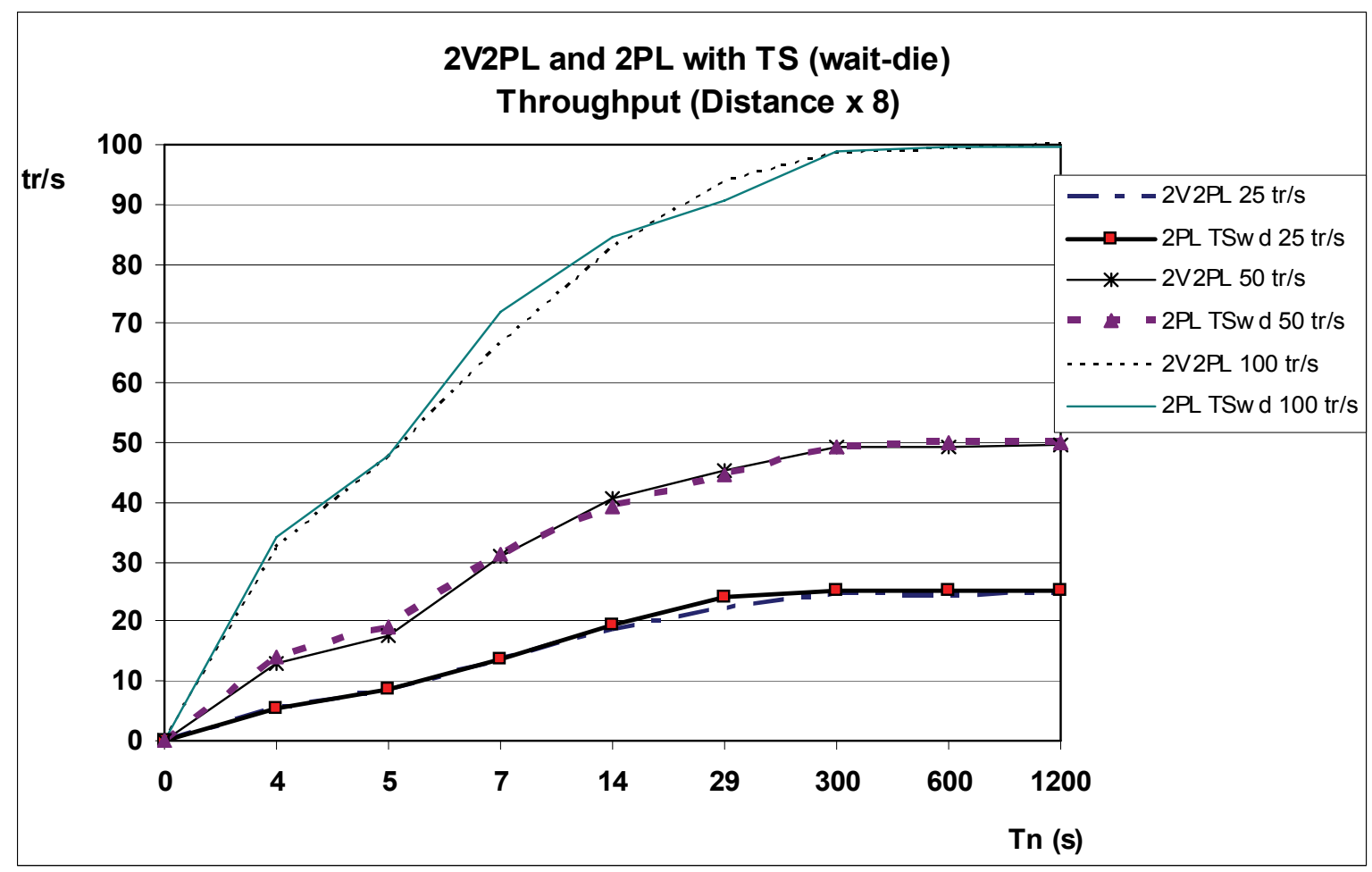

Figure 8. Throughput of the system (distance x 8) 


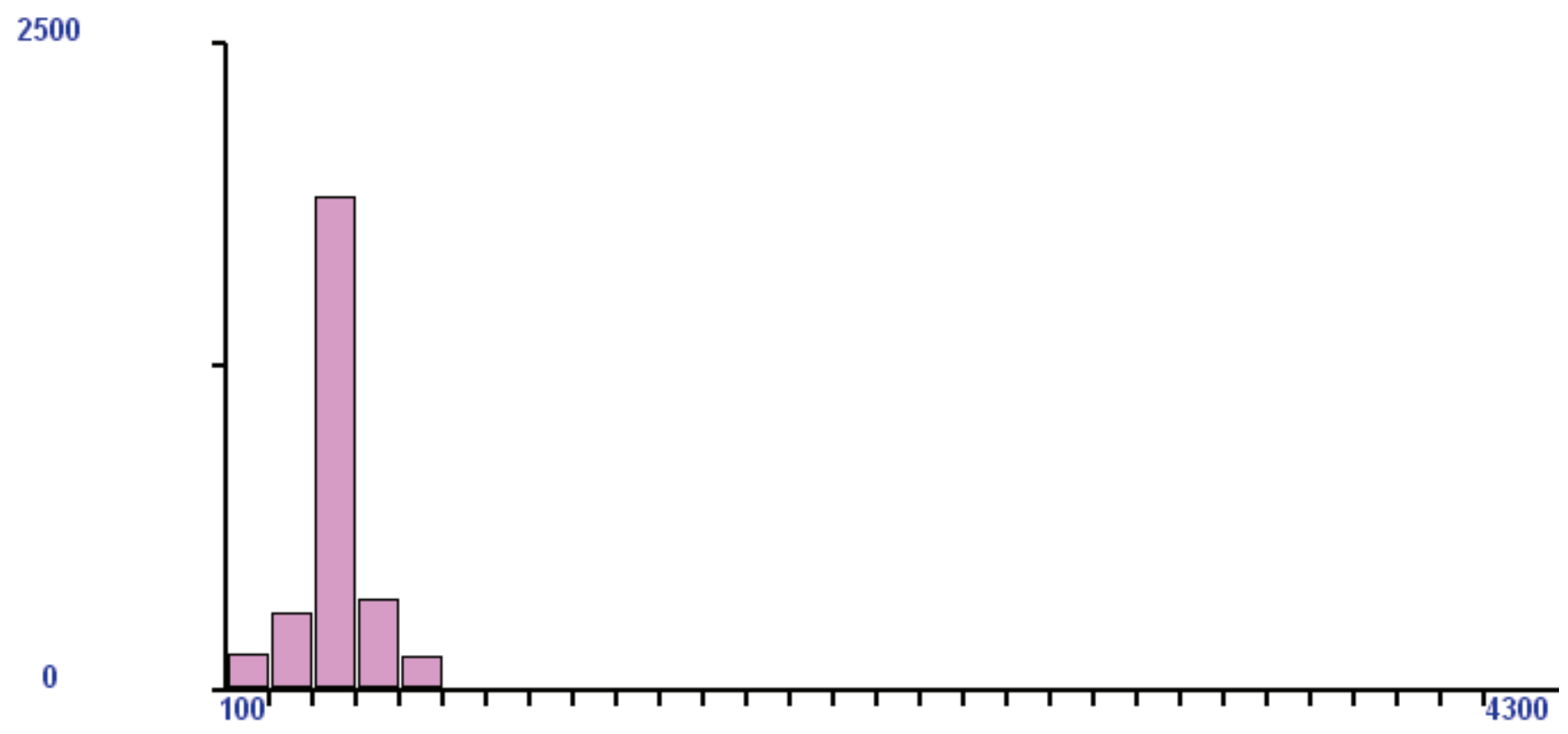

Figure 9. Frequency Distribution of RT in modeling Distributed 2V2PL at $\lambda=100 \mathrm{tr} / \mathrm{s}$

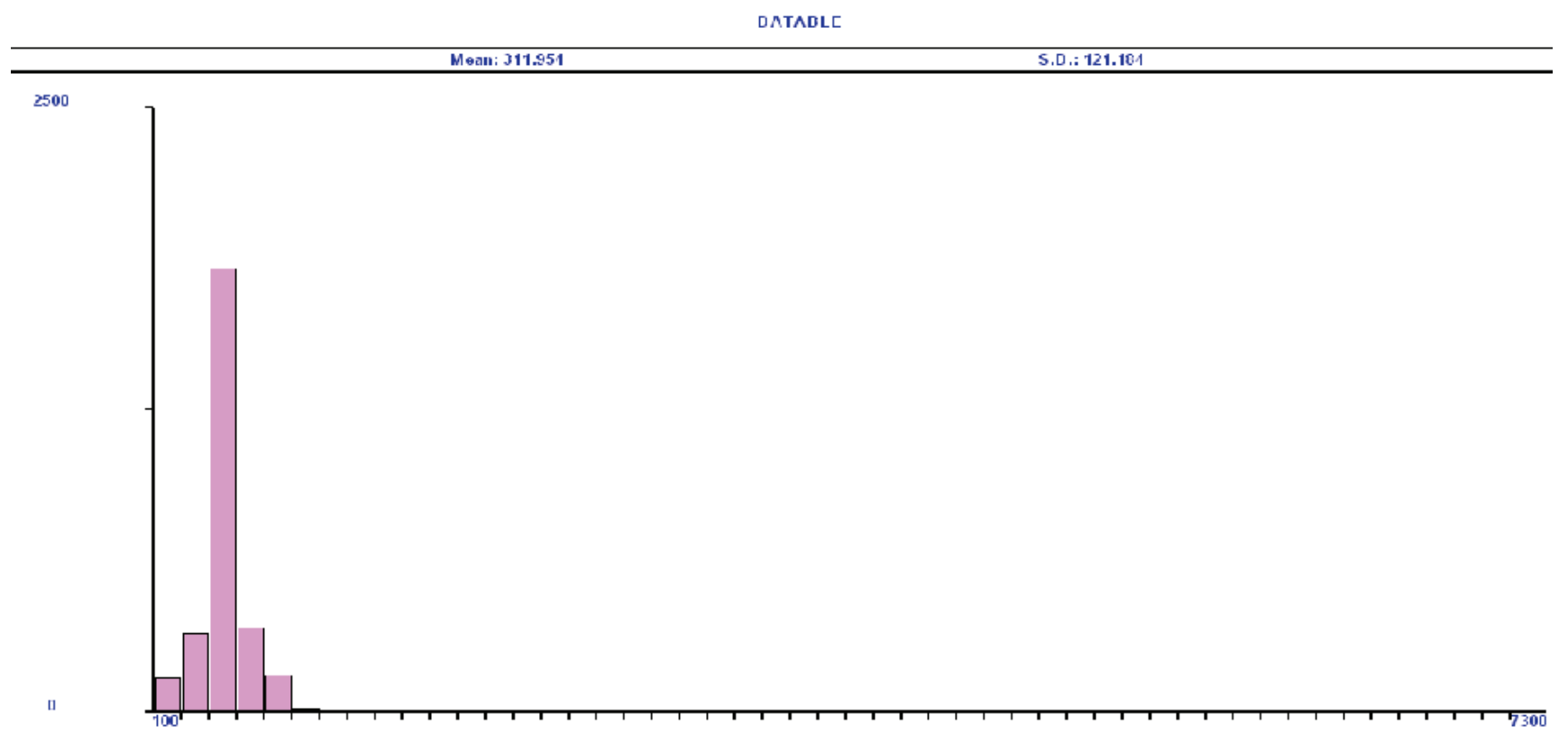

Figure 10. Frequency Distribution of RT in modelling Distributed 2PL with TS (wait-die) at $\lambda=100 \mathrm{tr} / \mathrm{s}$ 


\section{Conclusion}

The system of simulation GPSS World permits creation of effective simulation models of transaction concurrency control (in particular models of Two-phase locking of transactions in DDBMS with one-version and two-version architecture of replicated data.

The synthesized simulation models of GPSS World for Distributed 2V2PL and for Distributed 2PL with built-in mechanisms of timestamping (strategy "wait-die") allow experimentation and accumulation of statistical data to conduct comparative analysis of algorithms.

The proposed simulation models of Distributed Twoversion 2PL and Distributed 2PL with timestamping in the DDB are convergent and give good results in the experimental research. The comparative analysis of the results for modelling one-version and two-version Distributed 2PL show lack of deadlocks. The graphs of the throughput, service probability and the Response time have similar results.

It could be seen and compared with the template graphics and charts for throughput of figure 3, figure 5 and figure 6.

\section{References}

1. Bernstein, Ph., V. Hadzilacos, N. Goodman. Concurrency Control and Recovery in Database Systems. Addison-Wesley Publishing Company, 1987.

2. Carey, M., M. Livny. Distributed Concurrency Control Performance: A Study of Algorithms, Distribution and Replication. Proceedings of the 14th VLDB Conference, Los Angeles, 1988, 13-25. 3. Chardin, P. Multiversion Data and Transaction Concurrency Control. Open Systems, No. 1 [online], 2005 (in Russian) http://citforum.ru/database/articles/multiversion.

4. Connolly, T., C. Begg. Database Systems. Addison-Wesley, 2002.

5. Garcia-Molina, H., J. Ullman, J. Widom. Database Systems: The Complete Book. New Jersey 07458, Prentice Hall, 2001.

6. Srinisava, R., C. Williams, P. Reynolds. Distributed Transaction Processing on an Ordering Network. Technical Report CS-2001-08, February 2001.

7. Tomashevskii, V, E. Zhdanova. Simulations in GPSS. Bestseller, Moscow, 2003 (in Russian).

8. TPC Council. TPC - C Benchmark. Standard Specification Revision 5.11. Transaction Processing Performance Council, February 2010, 76-78.

9. Vasileva, S., A. Milev. Two-phase lock Modeling Algorithms Using Timestamp Ordering In Distributed Databases. International Conference Automatics and Informatics'11, 2-6 October 2011, Sofia, Bulgaria, B-359-B-362.

10. Vasileva, S., Y. Noskov. Simulation of Distributed Two-version Two-phase Locking. Fourth All-Russia Scientific-Practical Conference on Simulation and its Application in Science and Industry "Simulation. The Theory and Practice" (IMMOD-2009), II, 2009, 98-103 (in Russian).

\section{Manuscript received on 27.01.2016}

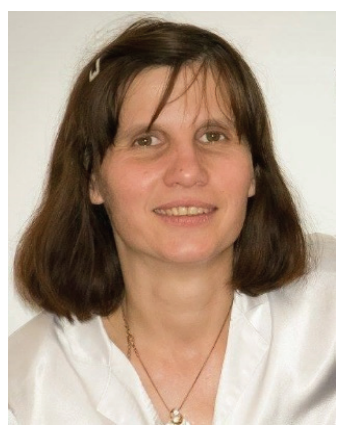

Svetlana Vasileva was born in Plodiv, Bulgaria and went to the Saint-Petersburg State Electrotechnical University (LETI), where she studied Engineer Process Systems "Computer Aided Design" and obtained her degree in 1994. She has also a degree as a PhD in Informatics. She worked as a programmer (1995-1996 - EF "DeytaSoft", Plovdiv), Assistant Professor of Informatics (1997-2000 Agricultural University, Plovdiv) before moving in 2001 to the University of Shumen, College Dobrich where currently she is a Chief Assistant Professor. Areas of scientific interests: control systems for distributed databases; simulation modeling of processes and systems; application of information technologies in education. Membership: John Atanasoff Society of Automatics and Informatics, Union of Scientists in Bulgaria, Bulgarian Modeling and Simulation Association - BULSIM, National Simulation Sosiety - Russia, IEEE and ACM.

Contacts:

Varna University of Management 9000 Varna, 13A Oborishte St. e-mail:svetlanaeli@abv.bg

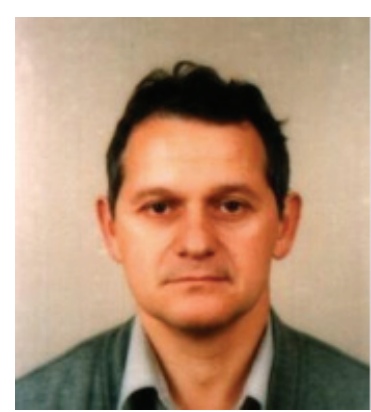

Aleksandar Milev was born in Shumen, Bulgaria. Prof. Aleksandar Milev, $P h D$ of Informatics works on simulation of systems and its application in networks. His research interests include communication systems, wireless communication technologies, operation systems and network security. Education details: Master of Science in Engineering (Military University "V. Levski”). Membership: John Atanasoff Society of Automatics and Informatics, Union of Scientists in Bulgaria.

Contacts:

Konstantin Preslavsky University of Shumen Faculty of Mathematics and Informatics 9712 Shumen, 115 Universitetska St. e-mail: alex_milev@yahoo.com 